\title{
De la célébration de la liberté de conscience à
} l'interprétation de la modernité

\section{Pierre-Olivier Léchot}

\section{(2) OpenEdition}

1 Journals

\section{Édition électronique}

URL : https://journals.openedition.org/assr/29732

DOI : $10.4000 /$ assr.29732

ISSN : $1777-5825$

Éditeur

Éditions de l'EHESS

\section{Édition imprimée}

Date de publication : 1 décembre 2017

Pagination : 179-189

ISSN : 0335-5985

\section{Référence électronique}

Pierre-Olivier Léchot, " De la célébration de la liberté de conscience à l'interprétation de la modernité », Archives de sciences sociales des religions [En ligne], 180 | octobre-décembre 2017, mis en ligne le 01 décembre 2019, consulté le 22 septembre 2021. URL : http://journals.openedition.org/assr/29732 ; DOI : https://doi.org/10.4000/assr.29732 


\title{
Pierre-Olivier Léchot
}

\section{De la célébration de la liberté de conscience à l'interprétation de la modernité}

\author{
À propos de: \\ ARnold Matthieu, Martin Luther, Paris, Fayard, 2017, 692 p. \\ Roper Lyndal, Martin Luther. Renegade and Prophet, Londres, Vintage, \\ 2017, 592 p. \\ SCHIlling Heinz, 1517. Weltgeschichte eines Jahres, Munich, C. H. \\ Beck, 2017, 363 p. \\ Wendebourg Dorothea, So viele Luthers... Die Reformationsjubiläen \\ des 19. und 20. Jahrhunderts, Leipzig, Evangelische Verlaganstalt, \\ 2017,294 p.
}

Que faisons-nous lorsque nous écrivons une biographie de Luther? Et qu'entendons-nous faire en nous intéressant à son œuvre, à sa pensée et à sa place dans l'histoire de la Réforme et de l'Europe ? Dans une conférence de 1924, préludant à son maître-ouvrage, Un destin. Martin Luther, paru en 1928, Lucien Febvre établissait avec une certaine fermeté le nécessaire préalable à une telle entreprise:

Il est nécessaire, pour le [Luther] connaître, de laver la crasse de trois siècles. Il faut débarrasser cette figure de Luther des immondices, des boues que les ennemis ont portées sur elle. Débarrassons aussi cette figure des couches de fard et de cosmétique que ses pieuses relations ont passées sur elle, pour l'embellir à leur façon. Débarrassons cette figure enfin des faux nez en carton que tant de gens bien intentionnés ont essayé de lui fixer - afin de moderniser Luther, de le tirer à eux ${ }^{1}$.

Lorsqu'il écrivait ces lignes, Febvre était encore confronté à nombre d'approches biographiques tenant plus du panégyrique ou, au contraire, de la dénonciation calomnieuse que de l'histoire au sens actuel du mot. Depuis lors, de telles lectures historiques sont devenues elles-mêmes objets d'étude et permettent de mettre à jour les projections d'une époque et les transformations subséquentes de ces dernières.

1. L. Febvre, "Pour comprendre Martin Luther ", présentation et notes de Pascale Gruson, Revue d'histoire du protestantisme, 2017-2, p. 200-201 


\section{Luther célébré}

C'est le mérite du très bel ouvrage de Dorothea Wendebourg, So viele Luther, que de le mettre en évidence ${ }^{2}$. Consacré aux célébrations du jubilé de 1517 en Allemagne aux $\mathrm{XIX}^{\mathrm{e}}$ et $\mathrm{XX}^{\mathrm{e}}$ siècles, le livre de l'historienne et théologienne allemande permet en effet de souligner combien Luther a pu représenter une réalité plastique lors des célébrations de l'affichage des 95 thèses ou de sa naissance ${ }^{3}$. Elle met ainsi en évidence le fait que Luther a pu, tour à tour, être célébré comme le champion de la liberté de conscience ou le héros de la nation allemande, le défenseur d'une réforme purement spirituelle du christianisme ou le précurseur de l'antisémitisme moderne. Constat surprenant si l'on se rappelle que l'époque concernée est celle qui voit le développement de l'historisme allemand et des méthodes historiographiques qui sont encore en bonne partie les nôtres: «Das Zeitalter des Historismus hat Luther und die Reformation kaum historisiert.» (p. 51). La raison en est pourtant évidente, pour peu que l'on se rappelle qu'il s'agissait aussi (et surtout) pour les protestants allemands de souligner la pertinence de Luther et, partant, de la confession protestante pour le présent de leur pays.

Ainsi, au fur et à mesure que se développent les thèses nationalistes, Luther se métamorphose sous la plume de ses biographes. En 1817, le théologien Friedrich Schleiermacher voit en lui non pas le précurseur des Lumières (ainsi que le font encore nombre de ses contemporains), mais le héros d'une vie religieuse authentique dans laquelle l'auteur des Discours sur la religion identifie la source des libertés fleurissant à son époque, ce qui l'autorise à conclure que célébrer la Réforme, c'est célébrer la liberté de la doctrine et de l'enseignement. Même constat, d'ailleurs, sous la plume d'auteurs juifs qui, quoique dans un esprit plus proche de celui des Lumières que de Schleiermacher, perçoivent en Luther le héros d'une liberté de conscience dont les juifs d'Allemagne ont pu largement bénéficier à sa suite. Il en va encore de même, un siècle plus tard, sous la plume du philosophe Hermann Cohen qui n'hésite pas à affirmer que tout juif peut se sentir «mit Luthers Glauben verbunden ", dans la mesure où l'insistance du Réformateur sur la foi, la grâce et le pardon fait écho aux piliers de la religion juive elle-même (p. 90-91).

La célébration de Luther à laquelle se livre Hermann Cohen apparaît cependant comme le dernier feu d'une époque en passe de disparaître. Si, lors du jubilé de 1883, le motif du Luther héros national ne semble pas encore dominant, il en va déjà bien autrement en cette année 1917 durant laquelle il prend la plume. Certes, les historiens qui entendent affirmer que Luther n'appartient pas qu'aux Allemands font encore entendre leur voix, malgré le premier conflit mondial - et ce ne sont pas les moins importants, à en juger par les propos de figures aussi telles que Karl Holl ou Ernst Troeltsch ${ }^{4}$; mais ils sont désormais minoritaires, la tendance

2. D. Wendebourg, So viele Luthers... Die Reformationsjubiläen des 19. und 20. Jahrhunderts, Leipzig, Evangelische Verlaganstalt, 2017.

3. Pour la France, on se référera au numéro spécial de la Revue d'histoire du protestantisme, codirigé par M. Arnold et P.-O. Léchot, «Le Luther des Français », 2017-2.

4. Il est du reste remarquable de constater que se joint alors à ces voix celle du théologien Emanuel Hirsch qui deviendra, sous le III ${ }^{e}$ Reich, l'un des plus fidèles soutiens du régime au sein de la communauté théologique protestante allemande. 
étant dès lors à la célébration d'un Réformateur ayant interprété le christianisme dans le plus pur esprit germanique, ainsi que le prétend un théologien comme Reinhold Seeberg. Nulle surprise, donc, que lors du jubilé de 1933, la tendance se confirme: Luther est désormais perçu, par exemple sous la plume de l'historien de l'Église Heinrich Bornkamm, comme l'incarnation de l'esprit allemand, par la suite appelé à se perpétuer sous l'impulsion des plus grands «chefs » de la nation, tels Bismarck ou Hitler lui-même (p. 161). Rares sont alors les voix qui détonnent dans ce concert nationaliste, même s'il convient de rappeler celles de certains partisans du mouvement théologique libéral allemand ou, de l'autre côté du spectre théologique, des défenseurs de la nouvelle théologie dialectique de Karl Barth.

Après guerre, bien sûr, l'image de Luther entame une nouvelle mutation et, en même temps, devient l'objet d'un conflit des mémoires, qu'on le célèbre en RFA ou en RDA. En République démocratique, Luther est d'abord perçu comme un traître à la cause prolétarienne lors du jubilé de 1946, en raison, bien évidemment, de son opposition à la révolte paysanne de 1525 , préfiguration des mouvements révolutionnaires des $\mathrm{XIX}^{\mathrm{e}}$ et $\mathrm{XX}^{\mathrm{e}}$ siècles. Mais bientôt, le ton change et les historiens comme les orateurs adoptent un point de vue plus dialectique qui reflète, d'ailleurs, celui de Marx lui-même: Luther apparaît sous leur plume comme le grand précurseur du mouvement de libération populaire, incarné dans toute sa pureté originelle par son contemporain et adversaire, Thomas Müntzer. Sans Luther, donc, pas de mouvement populaire, comme il ne peut y avoir de révolution prolétarienne sans révolution bourgeoise préalable. Notons cependant que dans pareille lecture la dimension religieuse de l'engagement de Luther ne joue plus aucun rôle. La raison de cette reprise positive de Luther, qui culmine en 1983 sous le régime Honecker à l'occasion des cinq-cents ans de la naissance du Réformateur, tient au fait que le second État allemand perçoit de plus en plus nettement la nécessité de définir sa place dans l'histoire nationale et a donc besoin de s'appuyer sur certaines figures tutélaires (p. 26). Il en est à certains égards de même en République fédérale où l'élan historiographique conduit à mettre en évidence les liens entre Luther et la Réforme d'une part et les sociétés urbaines et bourgeoises d'autre part, ceci afin de souligner le lien intrinsèque entre la culture démocratique de la République de Bonn, héritière sur ce point des villes d'empire, et un protestantisme allemand désormais débarrassé de ses oripeaux nationalistes. Il est d'ailleurs important de souligner qu'avant même la chute du Mur, les historiens des deux Républiques se rapprocheront pour prendre en compte les enseignements de ceux de l'autre camp et aboutir ainsi à l'actuelle historiographie allemande de la Réforme, soucieuse à la fois d'histoire sociale et d'histoire religieuse. La conclusion de Wendebourg s'impose donc d'elle-même et rejoint celle de Lucien Febvre: comprendre Luther, c'est d'abord l'historiciser, c'està-dire le tenir à distance, tout en ayant soin de connaître les interprétations dont il a pu être l'objet afin d'éviter de retomber dans leurs ornières.

\section{Luther historicisé}

C'est ce qu'ont tenté de faire, ces dernières années, trois biographies de Luther particulièrement stimulantes et qui, selon nous, se détachent des très et sans doute trop nombreuses contributions qui circulent sur le marché à l'occasion des cinq cents ans de la Réforme allemande. 
Parmi ces trois ouvrages, il faut d'abord mentionner celui de l'historienne oxonienne Lyndal Roper ${ }^{5}$ : résultat de plusieurs années d'intenses recherches, y compris dans les archives allemandes, le livre de Roper est magnifiquement écrit et se lit un peu comme un roman. Mais il a surtout le mérite de proposer de nouvelles pistes d'interprétation de la figure du moine saxon: sur le contexte de la famille de Luther, et en particulier sur le milieu minier saxon, mais également à propos des amitiés du Réformateur en dehors des cercles religieux et humanistes, dans le monde du commerce notamment - ce qui permet d'éclaircir certaines orientations de sa pensée et de son action. Parmi ces pistes, ce qui est tout particulièrement frappant, c'est la lecture que propose Roper de la relation, complexe mais instructive, de Luther au corps et à la sexualité ${ }^{6}$. Le caractère désinhibé du rapport de Luther à la sexualité est ainsi interprété par l'auteure comme la conséquence, paradoxale, de son augustinisme (p. 273) et, plus largement, de sa théologie de la grâce. Pour Luther, aucune œuvre humaine n'est bonne en dehors de la foi; par conséquent, le sexe, considéré comme un péché, n'est pas plus grave qu'un autre péché. En outre, compte tenu des thèses de Luther sur le serf arbitre, il est évident que la chasteté ne peut être l'objet de la volonté, toute dominée comme elle l'est par les affects. On ne peut donc reprocher à l'être humain son appétence pour la sexualité et ce d'autant moins que le sexe constitue une réalité naturelle, voulue par Dieu dans le but de la procréation (p. 295) - et, en ce sens, sa pratique ne peut être jugée que "saine", au sens médical du terme, selon le Réformateur (p. 297). L'acte sexuel apparaît ainsi à Luther comme tout aussi nécessaire, voire plus nécessaire que le fait de dormir, de manger ou de boire. Les prises de position du théologien saxon le conduisent ainsi à défendre la nécessité pour les chrétiens d'assumer leur corps - avec cette seule restriction: la liberté chrétienne doit être gouvernée en ces matières par l'amour et le respect du prochain. Certes, la vision de la sexualité et en particulier de la femme en tant qu'objet du désir de l'homme que développe Luther, en particulier dans ses Propos de table, demeure souvent très éloignée de celle qui prévaut de nos jours dans les sociétés occidentales; il n'en reste pas moins que, pour Roper, Luther contribua largement à dédramatiser la perception du sexe au sein de la société chrétienne d'Occident.

Le problème principal de cette première biographie tient cependant au fait que son auteure renoue selon nous de manière assez regrettable avec la lecture psychologisante de Luther jadis proposée par le psychanalyste Erik H. Erikson ${ }^{7}$, spécialiste de la révolte adolescente, auquel elle donne d'ailleurs crédit (p. 48): c'est dans la relation à ses parents qu'il faut ainsi chercher, selon elle, les causes principales des orientations théologiques de Luther, en particulier concernant sa vision de la foi et de Dieu. Le père, Hans Luder, c'est la figure divine menaçante

5. L. Roper, Martin Luther. Renegade and Prophet, Londres, Penguin Books, 2016.

6. De ce point de vue, il convient de signaler les recherches, également stimulantes, de Susan KarantNunn; voir par exemple: «Une œuvre de chair: l'acte sexuel en tant que liberté chrétienne dans la vie et la pensée de Martin Luther ", in O. Christin et Y. Krumenacker (éds.), Les protestants à l'époque moderne. Une approche anthropologique, Rennes, Presses universitaires de Rennes, 2017, p. 467-485.

7. E. H. Erikson, Young Man Luther: A Study in Psychoanalysis and History, New York, W. W. Norton \& Company, 1958 (trad. française sous le titre de Luther avant Luther. Psychanalyse et histoire, Paris, Flammarion, 1968). 
(p. 48) et le Dieu aimant et miséricordieux que découvre le jeune moine révolté lors de son «tournant réformateur ", c'est le père de substitution d'un Luther en rupture avec son père biologique à la suite de son entrée au couvent. Quant à la mère du Réformateur, c'est à elle, probablement, que Luther doit son insistance sur la dimension «concrète» et affective d'une théologie de l'expérience tournée vers les réalités concrètes de l'existence. On voit bien les limites de pareille approche: s'il est difficile, pour un thérapeute, de saisir les élans profonds de son patient alors qu'il est en mesure de le questionner directement, il est bien clair qu'il est encore plus délicat de répondre à cette question au sujet d'un auteur mort il y a près de cinq siècles et dont les représentations et l'univers mental étaient très éloignés des nôtres. C'est du reste la seconde faiblesse de cette biographie: celle-ci manque en effet d'une mise en contexte plus large de Luther: l' "homme Martin Luther " ${ }^{8}$ est en effet son centre d'intérêt quasi-exclusif - et l'on peut donc à bon droit se demander si la compréhension historique de la Réforme en tant que moment déterminant de l'histoire religieuse européenne y gagne réellement.

De ce point de vue, la biographie du meilleur spécialiste français de Luther, Matthieu Arnold, semble de loin plus utile au lecteur intéressé par cette dimension. Elle est le fruit de recherches de longue haleine que l'auteur a débutées par la publication, voici plus de vingt ans, de sa thèse sur la correspondance de Luther. La biographie de Matthieu Arnold n'est pas une biographie à thèse, comme l'est celle de Roper: elle ne fait «que» se concentrer sur les sources du temps, en tenant à une distance raisonnable les interprétations qui ont pu avoir cours par le passé - non sans en tenir compte à certaines occasions, surtout lorsqu'il s'agit de trancher un débat historiographique, ce qui, concernant Luther, n'est pas une mince affaire. En outre, et contrairement à de nombreuses biographies francophones, comme celle de Lucien Febvre en particulier, Matthieu Arnold à fait le choix de ne pas s'arrêter en 1525, alors que Luther est au sommet de sa carrière réformatrice, mais a entendu le suivre jusqu'à la fin de sa vie. Ce choix implique naturellement bien des difficultés que Matthieu Arnold surmonte avec brio, en particulier lorsqu'il n'hésite pas à aborder les questions les plus délicates avec un regard aussi honnête que précis (liens de Luther avec les pouvoirs politiques, relation au judaïsme, à l'islam, etc.).

La biographie de Matthieu Arnold a également pour elle un autre grand avantage $^{9}$ : contre bien des tendances historiographiques récentes, elle se veut un livre d'histoire religieuse, d'histoire de la théologie même, mais sans pour autant laisser de côté les acquis de l'histoire sociale, de l'histoire culturelle et de l'histoire des pratiques religieuses. La différence entre les historiens de la Réforme marqués par leur enracinement dans une faculté de théologie et les historiens issus des facultés de lettres ou de sciences humaines (les «Allgemeinhistoriker» comme disent les allemands) n'apparaît plus, sous sa plume, que comme une affaire de rattachement institutionnel. Il est heureux que cette opposition, souvent stérile, soit ici dépassée avec autant de sagacité. Quant à l'image de Luther, elle est désormais bien plus subtile et nuancée qu'au moment où Lucien Febvre prenait la plume pour dénoncer

8. Selon le titre de la version allemande du livre, parue simultanément à celle de l'édition anglaise: Der Mensch Martin Luther. Die Biographie, Francfort, S. Fischer, 2016.

9. M. Arnold, Martin Luther, Paris, Fayard, 2017. 
les lectures par trop biaisées de ses apologues ou de ses détracteurs: à la lecture de la biographie de Matthieu Arnold, il apparaît clairement que Luther ne peut plus être tenu pour ce héros de la conscience jadis dépeint par l'historiographie protestante, mais doit surtout être compris comme le défenseur d'un message de réconfort, un pasteur d'âmes, et un intellectuel créatif, mais aussi un caractère souvent emporté, rancunier et vindicatif.

Est-ce à dire, pourtant, que nous ne pouvons plus interpréter Luther pour notre temps - ou, pour le dire autrement, tenter de voir en quoi notre univers culturel et religieux lui est redevable? La réponse à cette question est donnée par la biographie de Luther, traduite en français, de l'historien berlinois Heinz Schilling ${ }^{10}$. Schilling partage avec Roper et, encore plus largement, avec Arnold un même souci de l'histoire qui le conduit à insister à maintes reprises sur la nécessité de tenir Luther à distance de nos représentations contemporaines. L'ouvrage s'ouvre ainsi sur une véritable leçon de méthode. Pour Schilling, Luther est l'homme "d'un monde qui n'est plus le nôtre et qui nous confronte à ce qui est inconnu et tout autre» (p. 15). Telle est, selon lui, la mission première de l'historien: mettre en évidence des réalités différentes des nôtres pour relativiser les contraintes herméneutiques du présent. Mais justement: sous la plume de Schilling, mettre à distance Luther conduit aussi, et précisément, à saisir plus finement ce qu'il a justement mis en route et qui nous conditionne encore - mais en distinguant justement «entre conséquences envisagées et conséquences non envisagées» (p. 16) ${ }^{11}$.

«Mettre à distance» signifie d'abord tenter d'inscrire la figure de Luther dans son horizon culturel au sens large du terme, à savoir « comprendre l'homme dans l'époque et l'époque dans l'homme ", selon l'expression de Gottfried Seebass rappelée en ouverture (p. 18). De la démarche de Schilling, il ressort que l'époque de Luther apparaît comme un temps de rupture ou, pour le dire en des termes moins abrupts, de transition fondamentale. Le temps de la Réforme protestante est en effet celui de la formation des empires mondiaux, avec la conquête du Nouveau Monde par les monarchies espagnoles et portugaises. Elle est aussi le temps d'un profond renouveau de la piété, d'une crise du sens aussi, tout en manifestant l'émergence, sous différentes formes, du capitalisme marchand, des sciences modernes, etc.

Il est évident, dans pareil contexte, que Luther ne plus être considéré comme l'initiateur de la modernité, de même que la Réforme n'en apparaît plus comme la matrice, tant il est vrai que la mise en lumière des transformations en train de s'opérer dans plusieurs champs de la culture européenne conduit à conclure que la modernité doit finalement bien peu à l'engagement réformateur de Luther.

10. H. Schilling, Martin Luther. Rebelle dans un temps de rupture, trad. de J.-L. Schlegel, Paris, Salvator, 2014; édition allemande: Martin Luther. Rebell in einer Zeit des Umbruchs, Munich, C. H. Beck, 2012. On notera, avec regret, que le traducteur n'ait pas entendu conserver les références historiographiques à la littérature autour de Luther, à laquelle l'édition allemande se réfère abondamment. Ce choix, approuvé de manière assez surprenante par l'auteur, a pour principal inconvénient qu'il est difficile pour le lecteur français de savoir à qui au juste Schilling fait référence lorsqu'il critique ou s'appuie sur une thèse particulière à propos de Luther.

11. Je me permets de mettre ici en évidence la proximité entre cette approche et ma propre démarche à l'occasion de mon "Que sais-je?»: P.-O. Léchot, La Réforme (1517-1564), Paris, Presses universitaires de France, 2017, en particulier p. 113-117. 
Cette vision des choses apparaît encore plus nettement dans l'ouvrage que Schilling vient de consacrer à l'année $1517^{12}$. Écrire l'histoire d'une année pour retracer les déplacements fondamentaux qui s'opèrent en un temps donné est une pratique historienne récente: on le retrouve par exemple dans le livre de Bartolomé et Lucile Benassar à propos de 1492 et de la découverte du Nouveau Monde ${ }^{13}$. Dans ce nouveau livre de l'historien allemand, Luther apparaît, dans sa radicalité religieuse, comme l'un des points de bascule d'une histoire en passe de devenir mondiale: en Europe, mais aussi en Afrique, en Asie et en Amérique, le monde change. 1517 n'est pas seulement, n'est pas d'abord l'année des 95 thèses, mais celle qui voit le sultan ottoman conquérir définitivement l'empire des Mamluks et s'ouvrir ainsi la porte de l'Afrique et de l'Arabie tout en récupérant, fait significatif, le titre de calife pour lui-même et ses descendants. 1517, c'est aussi l'année où, pour la première fois, des explorateurs portugais entrent en contact avec la cour de l'Empire chinois. En cette même année enfin, une ambassade est envoyée par le pape vers Moscou pour tenter de forger une alliance entre christianismes occidental et oriental contre la menace turque. Or, ces différentes dimensions, ces différents horizons sont en passe d'entrer en rapport et d'ouvrir ainsi une nouvelle ère: celle de l'histoire planétaire qu'il s'agit de comprendre, selon Schilling, non comme l'ère de l' " européisation» du monde mais bien en tant qu'un temps d'appropriations culturelles réciproques. L'étranger devient ainsi le propre d'une culture (p. 309). L'exemple du célèbre rhinocéros de Dürer est parlant de ce point de vue (p. 146-148): avec la représentation par Dürer de cet animal exotique rapporté des Indes à la cour du Portugal, la créature étrangère qu'elle était encore au début du XVI ${ }^{\mathrm{e}}$ siècle est progressivement passée dans la culture occidentale au point de lui appartenir pleinement - ce qui, au passage, permet à Schilling de relativiser quelque peu l'insistance de nombreux historiens sur l'irréductibilité culturelle des soi-disant vertus «occidentales». De ce point de vue, 1517. Weltgeschichte eines Jahres n'est pas tant un livre sur l'année 1517 qu'un ouvrage sur le début des temps modernes au même titre que sa biographie de Luther.

La conclusion de cette dernière le montre à l'envi. Certes, l'entreprise de Luther doit à bien des égards être lue comme un échec: de sa tentative de réforme universelle de la chrétienté éclot un monde qui voit celle-ci se fragmenter en États territoriaux luthériens, calvinistes et catholiques - mais justement: la fin de la prétention à l'universalité, même si elle fut maintenue sous la forme d'une «fiction» (p. 663) par le catholicisme post-tridentin, est un des points par lesquels Luther fit, à son corps défendant, entrer l'Europe dans la modernité religieuse, celle du pluralisme, en activant la différenciation de l'État et de la société. Dans le domaine politique, l'émergence des États confessionnels permit à ces derniers de prendre la main sur des réalités jusque-là relevant du domaine exclusif de l'Église, qu'il s'agisse du soin des pauvres, du contrôle et de la réglementation du mariage, de l'éducation et de la formation - toutes choses qui constituent de nos jours l'apanage des États modernes. Mais, en même temps, la résistance de Luther au pouvoir politique de Charles Quint peut être lue comme le commencement d'une nouvelle ère durant laquelle il devint de plus en plus possible et légitime de

12. H. Schilling, 1517. Weltgeschichte eines Jahres, Munich, C. H. Beck, 2017.

13. B. Benassar, L. Benassar, 1492. Un monde nouveau?, Paris, Perrin, 1991. 
résister, au nom de sa foi, à une autorité politique jugée tyrannique. De ce point de vue, le pluralisme et la liberté de conscience moderne peuvent être interprétés comme des conséquences de la Réforme protestante. Non pas qu'elles aient été voulues par Luther, bien au contraire, mais parce qu'en affirmant tout à la fois les droits de la conscience (encore, chez le Réformateur, soumise à la Parole de Dieu) et du sacerdoce universel de tous les croyants, Luther permit, contre son gré, que ce soit finalement l'individu qui décide en dernière instance de sa foi. Ne serait-ce que parce qu'aucun des deux camps, catholique ou protestant, ne parvint à s'imposer, il devint inévitable que les individus se prononcent en lieu et place des autorités confessionnelles et ce d'autant plus qu'ils y étaient encouragés, même indirectement, par les thèses de Luther sur la foi et la conscience, bientôt reprises et diffusées à travers toute l'Europe.

Là où, en revanche, l'influence de Luther sur la modernité fut la plus directe et sans doute la plus déterminante, ce fut dans sa "redécouverte de la religion et de la foi en tant que forces autochtones autant pour l'individu que pour la société » (p. 682). Ce faisant, la religion devient une puissance exerçant une influence bien plus grande, paradoxalement, que du temps de la papauté toute puissante. En outre, en insistant avec force sur la réalité quotidienne, sur l'importance de l'ordinaire comme lieu de réalisation de la vocation première du chrétien, Luther ne contribua pas tant au désenchantement du monde (contre Weber) qu'à une «mondanisation» de la religion. Du coup, toute sphère autrefois caractérisée comme mondaine en regard de celle, privilégiée, du clergé, devint le lieu même d'engagement du chrétien et de la réalisation de sa foi (mariage, famille, économie, politique, etc. $)^{14}$ :

En renforçant la religion comme une force originelle, non dérivée, vouée ni à la philosophie ni à l'art, en lui assignant le monde comme champ de son action et de son attestation, Luther libéra une dynamique qui apporta une contribution essentielle pour la transformation séculière de l'Europe au début des Temps modernes et, à plus long terme, pour l'advenue de la modernité. (p. 684).

\section{Luther interprété}

Sur la base de ces quelques lectures, nous pouvons tenter de répondre à notre question initiale: s'intéresser à Luther, c'est d'abord s'y intéresser parce que l'on mesure bien qu'avec lui se passe quelque-chose d'important pour le devenir de la modernité religieuse. L'intérêt des historiens pour la figure du moine saxon et pour son temps souligne en effet que Luther attire, questionne et donne à penser : il est, en somme, une figure qui interpelle - non pas tant en raison de ses affirmations théologiques qu'à cause, précisément, de ce qu'elles ont pu entraîner, fût-ce à l'opposé de l'intention de leur auteur. Or, comprendre en quoi Luther nous interpelle encore aujourd'hui passe, paradoxalement mais précisément, par le fait de le tenir à distance. Pour reprendre l'expression d'Ernst Troeltsch, il s'agit de «toujours à nouveau réduire en ruines l'histoire mythique et conventionnelle, lorsqu'elle s'est formée, pour retrouver comme Antée le contact avec le sol maternel de son

14. Cf. P.-O. Léchot, op. cit., p. 113-117. 
devenir. ${ }^{15}$ "Réduire en ruines » l'histoire mythique de Luther, c'est précisément le comprendre dans son temps, dans son contexte culturel, social et politique immédiat et plus global à la fois - c'est donc, d'abord, le considérer comme un "étranger», pour reprendre le terme de Heinz Schilling.

Mais c'est aussi, justement, s'autoriser ensuite à mieux percevoir en quoi sa pensée a en quelque sorte fécondé la modernité, que ce soit directement ou, le plus souvent, indirectement et involontairement. Or, il faut ici remarquer qu'il y a encore à ce sujet bien du travail pour les historiens: le cheminement, le déplacement et la mutation des idées de Luther, de sa cellule de travail à Wittenberg, jusque dans la culture dite «moderne» est un parcours complexe qui mérite que l'on y revienne. D'abord parce que la définition de la modernité est un sujet qui s'est passablement complexifié depuis l'époque où Max Weber et Ernst Troeltsch s'y sont intéressés. Ensuite, parce que l'objectif d'une telle étude se doit d'être précisé: il ne s'agit pas tant, ce faisant, de chercher à montrer en quoi notre société est "devenue protestante », comme le prétendent, parfois avec une fine intelligence, certains commentateurs contemporains ${ }^{16}$, que de saisir précisément ce qui se joue dans le rapport moderne au religieux, y compris là où nous l'attendons le moins. De ce point de vue, les apports des recherches récentes, comme celles de Heinz Schilling mais aussi de nombreux autres historiens, soulignent un fait important: la Réforme n'instaure pas une rupture avec le monde médiéval, pas plus qu'elle ne demeure en parfaite continuité avec ce dernier. L'émergence des temps modernes, comme le souligne Hans Blumenberg, est avant tout affaire de déplacements, de réinterprétations et de transitions ${ }^{17}$. Mais, au cœur de ces déplacements et de ces transitions, Luther joue, le plus souvent à son corps défendant, un rôle notoire.

Les quelques éléments que nous avons mis en évidence dans cette note critique nous orientent en effet vers une réalité centrale que soulignait naguère Bernard Cottret et que confirme l'étude de Heinz Schilling: c'est probablement dans la foi telle que refondée par Luther (Schilling parle, lui, de "religion»), c'est-à-dire en tant que puissance de mise en mouvement des grandes entreprises humaines, qu'il s'agit de chercher l'un des éléments moteurs des changements qui président à l'émergence du monde moderne ${ }^{18}$. Pour Luther, ce n'est plus, en effet, par la participation à des actes, mais bien par le biais d'une conviction personnelle, individuelle et subjective que le croyant est amené à entrer en relation avec son Dieu. Cette nouvelle orientation de la piété vers le for interne de la conscience engagera le développement de nombreux courants nouveaux. Elle inoculera éga-

15. Der Historismus und seine Probleme (1922), Berlin, De Gruyter, 2008, p. 1042 (Kritische Gesamtausgabe, t. 16/1 et 16/2), cité d'après H. Joas, Comment la personne est devenue sacrée. Une nouvelle généalogie des droits de l’homme, trad. de J.-M. Tétaz, Genève, Labor et Fides, 2016, p. 196.

16. Cf., par exemple, M. Alizart, Pop Théologie, Paris, Presses universitaires de France, 2015. 17. H. Blumenberg, La légitimité des temps modernes (1969/74), trad. de J.-L. Schlegel, Paris, Gallimard, 1999. Si la thèse blumenbergienne d'une origine non-protestante de la modernité est sujette à débat (voir à ce sujet: P. Büttgen, "Certitude et affirmation. La Réforme blumenberg ", Revue de métaphysique et de morale, 73, 2012-1, p. 35-51), son approche méthodologique me semble demeurer extrêmement stimulante.

18. B. Cottret, "La religion de Cromwell. Pour une histoire de la foi ", Historiens et géographes, Histoire religieuse I, Tiré à part n³41 (octobre 1993), p. 157-164. 
lement bien des ferments de division à une chrétienté jadis unifiée sous la houlette de la papauté, mais lui imposera également, du même coup, la reconnaissance d'une logique de pluralité religieuse: désormais, la foi sera d'abord l'affaire de l'individu, la fides qua prédominant résolument sur la fides quae, l'acte de foi sur le contenu de cette foi. S'intéresser à l'histoire de la foi, c'est donc non pas tant, d'abord, se concentrer sur l'histoire de la foi en tant que définie par les théologiens modernes (encore que cette dimension mérite toute notre attention), mais sur la foi en tant que réalité existentielle vivante, en tant que conviction personnelle, individuelle et subjective pouvant bien évidemment porter sur des réalités qualifiées instinctivement de "religieuses » mais aussi, précisément, sur des domaines considérés comme parfaitement "mondains" - conséquence, bien sûr, de cette «mondanisation» de la foi que signale Schilling. De ce point de vue, et pour le dire d'un trait, la question de la foi, c'est la question par excellence de la modernité.

Car ne nous y trompons pas: ce n'en est pas fini de la religion - ou de la foi. Le processus de «sortie de la religion » si souvent annoncé par certains historiens reste une réalité difficile à saisir de même que la notion, hautement complexe et bien trop souvent galvaudée, de "sécularisation " ${ }^{19}$. Comprendre le présent et les problèmes que "le religieux» y pose passe donc par une reprise de la question centrale qui agita naguère les historiens et les sociologues: comment les «chambardements» (Fernand Braudel) religieux du XvI ${ }^{\mathrm{e}}$ siècle accouchèrent-ils de la modernité ? Ainsi que le relèvent certains auteurs contemporains, il y a sans doute lieu, en effet, de rompre avec la thèse weberienne du désenchantement du monde pour saisir combien, y compris dans le «culte» contemporain des droits de l'homme par exemple ${ }^{20}$, se joue quelque-chose qui est bien de l'ordre du sacré ${ }^{21}$.

Resterait à saisir quel est précisément le lieu de cette nouvelle sacralité: est-ce la société ou l'individu ? Et si c'est l'individu, est-ce un individu concentré sur son intériorité et son autonomie subjective (Charles Taylor) ou bien un individu qui, au contraire, s'affirme (Hans Blumenberg) ? Si la sécularisation doit s'entendre, comme le suggère Jean-Claude Monod, comme neutralisation des visées religieuses d'universalisation du monde et de l'humain "en vue de la constitution d'une cité favorisant la détermination la plus libre possible des formes de vie et de croyances » 22 , il est clair, alors, que c'est au nom, justement, d'une valeur incommensurable accordée à la réalité individuelle de la croyance et à sa puissance de transformation de la réalité par l'action qu'il s'agit de comprendre les élans modernes en faveur des valeurs contemporaines. Cela étant dit, la thèse blumenbergienne d'une modernité comprise comme affirmation de soi d'un homme laissé à lui-même au sein d'un cosmos qui ne lui est plus destiné par un créateur bienveillant, à supposer que Blumenberg ait raison, n'est pas contradictoire, en soi, avec ce que nous venons de dire. Resterait toutefois à saisir le lien entre affirmation de soi et affirmation

19. Cf. J.-C. Monod, La querelle de la sécularisation, de Hegel à Blumenberg, Paris, Vrin, 2012. 20. Cf. V. Zuber, Le culte des droits de l'homme, Paris, Gallimard, 2015 et Id., Les origines religieuses des droits de l'homme. Le christianisme face aux libertés modernes (XVIII ${ }^{e}-\mathrm{XXI}^{e}$ siècles), Genève, Labor et Fides, 2017.

21. Cf. H. Joas, op. cit., et, plus récemment, Id., Die Macht des Heiligen. Eine Alternative zur Geschichte von der Entzauberung, Berlin, Suhrkamp, 2017.

22. J.-C. Monod, op. cit., p. 291. 
de la foi ${ }^{23}$ ce qui, compte tenu de la propension réformatrice puis protestante à la rédaction de formulaires de foi collectifs et individuels, n'est pas sans lien avec la thématique que nous avons tenté d'expliciter rapidement dans ces lignes.

Pierre-Olivier LÉCHOT

Institut protestant de théologie - Faculté de Paris pierre-olivier.lechot@iptheologie.fr

23. Cf. P. Büttgen, art. cité. 